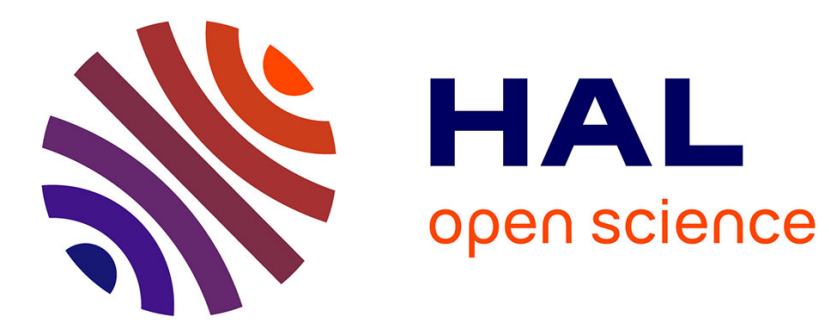

\title{
L'effet de la fréquence lexicale sur les réalisations des rhotiques en Ecosse
}

\author{
Monika Pukli
}

\section{To cite this version:}

Monika Pukli. L'effet de la fréquence lexicale sur les réalisations des rhotiques en Ecosse. XXXIIe Journées d'Études sur la Parole, 2018, Aix en Provence, France. hal-02530429

\section{HAL Id: hal-02530429 \\ https://hal.science/hal-02530429}

Submitted on 2 Apr 2020

HAL is a multi-disciplinary open access archive for the deposit and dissemination of scientific research documents, whether they are published or not. The documents may come from teaching and research institutions in France or abroad, or from public or private research centers.
L'archive ouverte pluridisciplinaire HAL, est destinée au dépôt et à la diffusion de documents scientifiques de niveau recherche, publiés ou non, émanant des établissements d'enseignement et de recherche français ou étrangers, des laboratoires publics ou privés. 


\title{
L'effet de la fréquence lexicale sur les réalisations des rhotiques en Écosse
}

\author{
Monika Pukli ${ }^{1}$ \\ (1) LiLPa EA 1339, 22 rue R. Descartes, 67100 Strasbourg, France \\ mpukli@unistra.fr
}

RESUME

Cet article présente les résultats d'une étude auditive et acoustique des réalisations du phonème $/ \mathrm{r} / \mathrm{en}$ position de coda dans l'anglais parlé en Écosse, avec comme objectif de tester le lien entre la fréquence lexicale et les formes non rhotiques. Bien que la lénition des rhotiques en Écosse partant d'une vibrante roulée et évoluant vers une approximante accompagnée de réalisations de plus en plus vocalisées devrait être sensible à la fréquence lexicale, les données relevées en parole spontanée chez dix-huit locuteurs ne montrent pas de corrélation significative entre celle-ci et les formes non rhotiques. Par conséquent, l'article propose de s'interroger d'une part sur la cohérence méthodologique, et d'autre part sur la possibilité d'interprétation de la fréquence à différentes échelles.

\section{ABSTRACT}

\section{Lexical frequency effects on rhotic realisations in Scotland}

This paper presents results from an auditory and acoustic study of coda /r/ realisations in Scottish English related to potential frequency effects on non-rhotic occurrences. Although lenition from the Scottish trill towards approximants and more vocalic realisations should be sensitive to word frequency, findings from spontaneous speech in 18 speakers do not show a significant correlation between non-rhotic forms and lexical frequency. Consequently, we raise questions on the coherence across methodological practices on the one hand, and on the interpretation of observed frequency effects on different levels, on the other.

MOTS-CLES : fréquence lexicale, théories basées sur l'usage, anglais écossais

KEYWORDS: lexical frequency, usage-based grammar, Scottish English 


\section{Introduction}

Cette étude s'interroge sur le rôle de la fréquence lexicale dans le changement phonologique dans le contexte de la dérhoticisation en Écosse de nos jours. La variation phonétique des formes rhotiques en anglais écossais, qui peut être conditionnée par des facteurs liés à l'usager et à l'usage, fait partie d'une lénition graduelle particulière car, mené à terme, le changement suppose une réinterprétation des contraintes phonotactiques. Cette lénition est déterminée par le profil sociolinguistique du groupe d'usagers ainsi que par les caractéristiques phonologiques du mot (y compris, en fonction du cadre théorique, la fréquence lexicale). C'est sur ce dernier point que l'étude acoustique et auditive se concentre ici.

En 1929, dans sa thèse intitulée Relative frequency as a determinant of phonetic change, Zipf a établi une corrélation entre certains changements phonologiques et le rang de fréquence du phonème dans la langue. Depuis, mais surtout à l'instar des propositions de Chen et Wang (1975), l'étude du changement s'intéresse tout particulièrement à la diffusion lexicale des formes innovantes et il semble que, selon le type de changement, l'actuation puisse toucher soit en premier lieu les mots fréquents, soit d'abord les mots rares. Au-delà d'une description fidèle de la diffusion des innovations dans la population, les approches sociocognitives ont également élaboré une représentation mentale basée sur la fréquence lexicale des items (phonèmes, morphèmes, collocations, etc.). Plusieurs modèles plus ou moins 'extrêmes' de la Théorie des Exemplaires se basent aujourd'hui sur l'hypothèse selon laquelle la fréquence d'un mot a une influence directe sur sa représentation mentale (voir Ernestus \& Baayens, 2011 pour un survol de ces modèles).

La dérhoticisation en Écosse fait référence aux résultats de différentes études qui mettent en relation l'âge des locuteurs et leur tendance à recourir à des réalisations vocaliques, voire absentes du phonème /r/ en position de coda. Même s'il n'existe à notre connaissance pas d'étude de véritable grande ampleur ${ }^{1}$, plusieurs enquêtes indépendantes ont démontré l'usage plus ou moins étendu de séquences non-rhotiques dans différentes villes d'Écosse, et les chercheurs s'accordent sur le fait qu'il existe une évolution tendant vers la perte de la rhoticité.

Du point de vue phonologique, ce processus peut représenter une évolution graduelle au niveau phonétique menant à un remaniement du système phonologique à terme (au contraire d'un changement abrupt, pour ceux qui souscrivent à cette dichotomie, où une variante remplace une autre sans réalisations intermédiaires). Le changement graduel peut être perçu dans des formes très hétérogènes, consonantiques et vocaliques, se situant sur un continuum de réalisations intermédiaires entre le [r] traditionnel qui est attesté encore aujourd'hui et l'absence totale du phonème. Mais surtout, la dérhoticisation peut également être conçue comme une lénition ayant pour origine cette vibrante roulée alvéolaire remontant à il y a plus de cent ans, passant par deux réalisations dominantes 'en compétition', vibrante battue vs. approximante, et arrivant à une réalisation entièrement vocalique ou zéro (Stuart Smith et al, 2014). Selon le point de vue adopté, on s'attend

Johntson (1997) examine notamment 91 locuteurs d'Edimbourg, et Jauriberry présente 161 locuteurs de cinq régions d'Ecosse. 
ainsi soit à une absence de l'effet de fréquence lexicale (changement graduel), soit au contraire à un lien fort entre fréquence et formes non rhotiques (lénition).

De notre point de vue, la perte de rhotiques consonantiques s'inscrit dans l'évolution de lénition et nous souhaitons donc tester l'hypothèse d'une corrélation entre forme non rhotique et mot fréquent. Ainsi, dans un schéma simplifié de la Théorie des Exemplaires, la lénition d'un segment dans la production quotidienne et répétée produite par un relâchement persistant de l'effort pour atteindre la cible articulatoire devrait induire un changement dans l'exemplaire, ce qui à son tour se manifesterait dans une production plus rapidement modifiée et donc plus répandue dans les mots fréquents.

\section{Corpus et méthodologie}

Les données analysées proviennent du corpus phonologique du programme PAC enregistré au début des années 2000. Dix-huit locuteurs écossais ont été étudiés en parole continue spontanée: huit hommes et dix femmes d'âges variant entre 18 et 82 ans, d'un milieu socio-économique allant d'ouvrier à ingénieur. Ils sont tous nés et ont grandi dans la ville d'Ayr même ou dans ses environs immédiats. La variation en matière de rhoticité a déjà été relevée chez certains locuteurs de ce corpus (Jauriberry et al, 2012) mais pas sur la totalité de l'échantillon.

Afin de tester l'effet potentiel de la fréquence lexicale sur les variantes phonétiques nous avons utilisé le corpus BNC, le corpus oral PAC de la variété locale étant trop petit. Le BNC, avec ses 100 millions de mots lui-même, n'est pas très conséquent par rapport à nos standards actuels, et pour cette raison les lemmes venant du corpus entier ont été pris en compte (Kilgarriff, 1998), mélangeant oral et écrit des années 1980-1990. Les noms propres (items orthographiés avec une majuscule) et les chiffres ont été ignorés, et la valeur forfaitaire 790 a été assignée aux mots avec moins de 800 occurrences dans le BNC.

Nous avons recueilli toutes les occurrences de /r/ en position de coda non suivi de voyelle dans les conversations enregistrées en équilibrant leur nombre entre locuteurs ayant un échange de 20 minutes et ceux qui parlent moins longtemps, c'est-à-dire entre 25 et 40 mots par sujet. Le repérage des occurrences a systématiquement été effectué à partir de la fin et vers le début de l'entretien. De ces données initialement retenues, nous avons enlevé les réalisations non utilisables à l'issue de l'analyse acoustique et les résultats sont ainsi basés sur 566 mesures.

Il est important de noter que les mots grammaticaux ne font pas partie de la sélection étudiée. En effet, ces mots ont une fréquence exceptionnellement haute mais sont articulés de manière très approximative dans les séquences sans accent en anglais. Leurs réductions prépondérantes, y compris naturellement celle du phonème $/ \mathrm{r}$, auraient potentiellement une influence sur les résultats, or les modifications dues au rythme du discours et au tempo de l'élocution constituent des effets qui touchent tous les phonèmes et pas uniquement $/ \mathrm{r} /$. Par ailleurs, certaines occurrences de mots lexicaux sont également affectées par un tempo très élevé ; ces réalisations de réduction de syllabe 
entière ont été retenues pour l'analyse (et sont donc autant d'occurrences de non-rhoticité dans nos résultats).

Les réalisations des rhotiques ont d'abord été analysées de manière auditive sur deux sessions par le même évaluateur (l'auteure). Une analyse acoustique des formants a ensuite été utilisée pour les occurrences où l'étude auditive s'est avérée non conclusive. Cette analyse a suivi les conventions de la littérature ${ }^{2}$ tout en acceptant les formes ambiguës comme étant rhotiques, compensant ainsi d'une part la pauvreté relative d'indices sûrs dans le spectre acoustique pour certaines des formes phonétiques que peuvent prendre les rhotiques en anglais, et d'autre part le nombre incertain d'occurrences ayant une articulation consonantique invisible sur l'image acoustique à cause de l'achèvement du geste lingual survenant après l'arrêt de la phonation (Lawson et al, 2008). Ce procédé représente un risque de surestimation de formes rhotiques qui nous semble être préférable au cas contraire. La non-rhoticité globale observée est ainsi de $42 \%$ des occurrences totales.

\section{Résultats et discussion}

Les variables retenues pour l'appréciation des résultats ont été les suivantes: la valeur de la fréquence lexicale (forme brute et plusieurs variables transformées), l'accentuation (syllabe accentuée vs. non accentuée), l'environnement segmental (position finale avant pause (anymore), position finale pré-consonantique (better than) et position médiane pré-consonantique (mermaid, escort)), ainsi que l'âge, le sexe et le statut socio-économique des locuteurs.

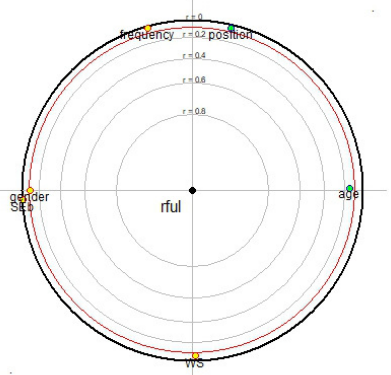

FIGURE 1: L'interaction entre variables, projetée par une analyse en composante principale focalisée (variables explicatives en partant de la gauche dans le sens de l'aiguille d'une montre: sexe, profil socio-économique, fréquence lexicale, position (avant pause ou non), âge, accent syllabique).

2 Pour les critères de segmentation des 14 formes rhotiques les plus courantes, voir Jauriberry (2016 : 218), pour une étude récente sur l'interaction entre acoustique, gestes articulatoires et perception de la structure formantique des rhotiques écossaises, voir Lawson et al (2018). 
Nous avons d'abord tenté d'étudier l'interaction des variables explicatives en vue de réaliser une régression logistique. L'analyse en composante principale focalisée (voir Figure 1 ci-dessus) effectuée par la commande $f p c a$ de la librairie $p s y$ avec $\mathrm{R}$ montre que les variables présentes dans notre tableau n'ont pas de corrélation positive entre elles. Au centre du cercle se trouve la rhoticité (la variable à expliquer) et nous nous intéressons à des points-variable regroupés dans l'image autour de la fréquence. On peut constater que la fréquence lexicale est bien-entendu indépendante des caractéristiques des usagers tel que l'âge, le profil socio-économique et le sexe. Par ailleurs, toutes les variables à l'exception de l'âge sont en deçà du cercle rouge et seront donc vraisemblablement non significatives (au seuil de 5\%).

Si l'on compare la distribution de la variable fréquence (données non transformées) avec celle de l'âge en fonction de la présence et de l'absence de /r/ (Figure 2 ci-dessous), on voit clairement l'effet de l'âge (à droite) alors que l'étendue de la fréquence lexicale des mots ayant des formes non rhotiques est très semblable à celle des mots rhotiques (à gauche).
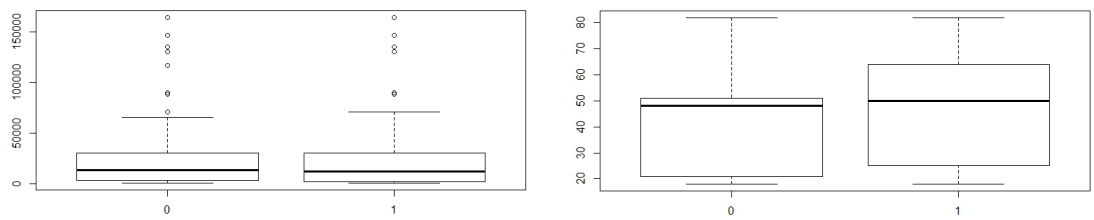

FIGURE 2: La distribution des formes non rhotiques (0) et rhotiques (1) selon la fréquence (à gauche) et selon l'âge (à droite)

Une régression logistique au seul effet de fréquence donne une valeur de $\mathrm{p}$ non significative aussi bien pour la fréquence dans sa forme brute $(\mathrm{p}=0.65922)$ que dans sa forme logarithmique $(\mathrm{p}=0.185)$ par rapport aux occurrences des formes non rhotiques. De même, lorsque les observations sont recodées avec une variable de rang de fréquence allant de 0.2 (le mot le plus rare dans le corpus, bard) à 208 (le mot le plus fréquent, year), il n'y pas de lien entre la (non) réalisation de $/ \mathrm{r} /$ et la fréquence de mot $(\mathrm{p}=0.20188)$. Les effets combinés de fréquence + accent lexical, et de fréquence + position de /r/ dans le mot ne sont pas significatifs non plus (respectivement 0.1188 et 0.227 ).

Le seul facteur phonologique ayant un lien avec la rhoticité est celui de l'accent lexical (X-squared = 5.8302 , df $=1$, p-value $=0.01575)$ : les formes non rhotiques apparaissent plus souvent dans les syllabes non accentuées. Il convient cependant d'ajouter qu'il s'agit d'un codage non empirique ; l'accentuation est notée selon la forme isolée du mot sur deux niveaux: absence vs présence d'accent.

Dans un second temps, la variable de la fréquence a été reformulée en variable catégorielle avec quatre valeurs allant de mot rare à très fréquent : 790-2935, 2936-12613, 12614-30594 et 30595- 
163930, correspondant aux quatre quartiles de la variable numérique. Cette fois-ci une corrélation légèrement significative émerge entre la catégorie de fréquence et la rhoticité des mots $(\mathrm{p}=0.04321)$ si l'on compare les mots rhotiques et non rhotiques.

Comment interpréter ces résultats ? D'abord se pose toujours la question de la méthodologie utilisée (et de l'échantillon sur lequel les mesures et calculs sont basés) : combien de locuteurs, combien de tokens, quel type de données (lecture de mot, lecture de phrase, lecture de texte, échange cadré par une activité précise, échange en présence ou non de l'enquêteur, etc. sont autant de facteurs ayant une influence possible sur les résultats), quelles analyses statistiques. A ces questions d'ordre général s'ajoutent celles en lien avec la fréquence :

- la variable de la fréquence lexicale est-elle établie avec le logarithme de la valeur, en valeur absolue, ou encore avec une recatégorisation en 'rare-moyen-fréquent' ? Quels sont les paramètres de la recatégorisation?

- la distribution de la fréquence lexicale des mots, connue pour être non normale, est-elle un facteur à prendre en compte dans l'analyse statistique ?

- la fréquence d'un mot donné provient-elle d'un corpus oral de la variété locale (et par conséquent plus petit) ou d'un corpus 'généraliste' de la variété ?

- la liste de fréquence de ce corpus de référence est-elle lemmatisée, et si non, comment les formes sont-elles comptées ?

- au-delà de la fréquence des mots, celle des phonèmes et des cooccurrences de phonèmes peutelle être pertinente?

Il est rare que tous ces choix et caractéristiques soient clairement documentés, et peu nombreuses sont les études pouvant être reproduites.

Puis, l'interprétation des résultats revient sur la nature du phénomène étudié. De notre point de vue, la perte de rhotiques consonantiques s'inscrit dans l'évolution de lénition décrite plus haut, or les résultats observés ne sont pas ceux attendus. Il est possible que l'effet de fréquence ne se montre que sur certaines échelles et que cela soit masqué dans l'effet global. ${ }^{3}$ La transformation des données brutes de la fréquence lexicale est nécessaire à cause de la distribution de la valeur qui suit une loi de puissance au lieu d'une loi normale. Mais la variable obtenue par transformation logarithmique n'est statistiquement pas liée à la rhoticité.

On ne peut pas passer ici en revue les études tentant à lier fréquence et rhoticité en anglais. A titre d'exemple, Nagy et Irwin (2010) ont trouvé un lien faible entre rhoticité et fréquence en anglais parlé à Boston, cependant, l'étude la plus pertinente sur l'anglais écossais (Jauriberry 2016) n'a pas observé d'effet de fréquence dans la variation des réalisations rhotiques. Cela est autant plus troublant que l'auteur a effectué une recatégorisation de la fréquence.

La représentation en exemplaires qu'offrent plusieurs modèles actuels est d'un niveau de complexité tel qu'un simple test empirique peut difficilement la valider. Il ne faut pas oublier l'hétérogénéité des

Je tiens à remercier un des relecteurs anonymes pour cette idée. 
facteurs à prendre en compte dans l'interprétation des résultats liés à la fréquence, et ce sur plusieurs niveaux de la représentation. A titre d'exemple, suivant Pierrehumbert (2006), l'effet de saturation et la non linéarité de la représentation du mot, l'effet de saillance résultant de l'encodage dans la mémoire, la dissociation de la perception et de la production, ou encore les effets liés aux réseaux complexes et la densité des connections. En d'autres termes une variable qui semble simple et direct ne l'est pas forcément.

\section{Conclusions}

Nous avons étudié les effets éventuels de la fréquence lexicale sur la dérhoticisation en Écosse et, au lieu d'un lien fort, nous avons trouvé une faible corrélation entre elles deux en comparant la rhoticité dans les quatre quartiles de la distribution totale des mots.

Pour une théorie phonologique sociocognitive, l'objectif est d'une part de modéliser l'usage de la langue dans la communauté, et d'autre part d'offrir une représentation mentale de la langue chez l'individu. Il n'est pas certain que les mêmes principes et processus puissent être utilisés dans les deux cas. Notre étude ne peut pas porter sur la diffusion des innovations avec un échantillon de dixhuit locuteurs même en faisant confiance à la puissance des tests statistiques. Il est aujourd'hui de plus en plus clair que l'étude du changement linguistique ne peut s'envisager que soit sur le niveau microscopique d'une étude ethnographique, soit sur un niveau beaucoup plus large avec des simulations computationnelles (Wagner \& Abtahian, 2016). Ainsi, les différents facteurs phonologiques, sociophonétiques et psychologiques ne sont pas en compétition pour 'mener' un changement linguistique mais sont tous potentiellement concernés. Le défi est de faire des simulations où les agrégats de ces facteurs sont établis précisément.

Compte tenu de nos résultats, nous nous interrogeons sur la manière dont on doit aborder l'étude du changement linguistique : par la diffusion des formes innovantes que l'on peut observer dans la communauté ou par la représentation de la parole dans le système phonologique de l'individu ? Si l'approche sociocognitive a pour objectif d'expliciter les deux aspects, encore faut-il justifier que la même méthode de représentation est applicable aussi bien chez l'individu pendant et après la période d'acquisition qu'au sein de la population. La variation présente de manière inhérente et constante dans un groupe est-elle inscrite réellement dans la représentation mentale de chacun des locuteurs ? Ce qui semble être certain, c'est que le lien entre la diffusion des innovations phonétiques et l'évolution de leur schéma cognitif chez l'individu n'est pas direct.

\section{Références}

CHEN M. \& WANG W.S-Y. (1975). Sound change: Actuation and implementation. Linguistics. 51. 255-8. 
ERNESTUS M. \& BAAYENS R. H. (2011). Corpora and exemplars in phonology. In GOLDSMITH J A, RIGGLE J \& YU A C. (Eds.) The handbook of phonological theory (2 $2^{\text {nd }}$ ed.) Oxford: WileyBlackwell.

JAURIBERRY T. (2016). Rhotiques et rhoticité en Ecosse : une étude sociophonétique de l'anglais écossais standard. Thèse de doctorat. Université de Strasbourg.

JAURIBERRY T., SOCK R., HAMm A. \& PukLi M. (2012). Rhoticité et dérhoticisation en anglais écossais d'Ayrshire. Actes de JEP. 89-96.

JOHNTSON P. (1997). Regional variation. In JONES C. (Ed.) The Edinburgh History of Scots, Edinburgh : Edinburgh University Press, 433-513.

KILGARRIFF A. (1998). BNC database and word frequency lists. En-ligne : https://kilgarriff.co.uk/bnc-readme.html.

LAWSON E., STUART-SMITH J. \& SCOBBIE J. (2018). The role of gesture delay in coda /r/ weakening: An articulatory, auditory and acoustic study. The Journal of the Acoustical Society of America. 143, 1646. doi/10.1121/1.5027833.

LAwson E., Stuart-Smith J. \& ScobbIE J. (2008). Articulatory insights into language variation and change: Preliminary findings from an ultrasound study of derhoticization in Scottish English. University of Pennsylvania Working Papers in Linguistics. 36, 102-110.

NAGY N. \& IRWIN P. (2010). Boston (r): Neighbo(r)s nea(r) and fa(r). Language Variation and Change, 22, 241-278.

PIERREHUMBERT J. (2006). The next toolkit. Journal of Phonetics 34(6), 516-530.

Stuart Smith J., Lawson E. \& Scobbie J. M. (2014). Derhoticisation in Scottish English: a sociophonetic journey. Celata C. \& CAlamaI S. (Eds.) Advances in Sociophonetics. John Benjamins, Amsterdam. http://eprints.gla.ac.uk/87460.

Wagner S. E. \& AbTahian M. R. (2016). Social Networks and the Study of Language Variation and Change. In NeAL Z. P. (Ed.) Handbook of Applied System Science.

ZIPF G. K. (1929). Relative frequency as a determinant of phonetic change. Harvard Studies in classical philology, $\mathbf{X L}$. 